\title{
ON AFFINE TRANSFORMATIONS OF A RIEMANNIAN MANIFOLD*
}

\author{
JUN-ICHI HANO
}

In this paper we establish some theorems about the group of affine transformations on a Riemannian manifold. First we prove a decomposition theorem (Theorem 1) of the largest connected group of affine transformations on a simply connected complete Riemannian manifold, which corresponds to the decomposition theorem of de Rham [4] ${ }^{1)}$ for the manifold. In the case of the largest group of isometries, a theorem of the same type is found in de Rham's paper [4] in a weaker form. Using Theorem 1 we obtain a sufficient condition for an infinitesimal affine transformation to be a Killing vector field (Theorem 2). This result includes K. Yano's theorem [13] which states that on a compact Riemannian manifold an infinitesimal affine transformation is always a Killing vector field. His proof of the theorem depends on an integral formula which is valid only for a compact manifold. Our method is quite different and is based on a result [11] of K. Nomizu.

The author expresses his deep thanks to Dr. Nomizu who suggested these problems to him.

\section{Preliminaries}

1. Let $M$ be a differentiable manifold of class $C^{\infty} .^{2)}$ The set $\mathfrak{I}$ of all tangent vector fields defined on $M$ is a module over the ring $\mathfrak{F}$ of all differentiable functions on $M$.

An affine connection is defined by a homomorphism over $\mathfrak{F}: X \rightarrow \nabla_{X}$ from $\mathfrak{I}$ into the module of linear mappings (over the field of all real numbers) of $T$, which satisfies the following condition

Received March 10, 1955.

* The subject of this paper was prepared while the author was a Yukawa Fellow at Osaka University.

1) Numbers in brackets refer to Bibliography at the end of this paper.

2) As we only consider manifolds, tangent vector fields, tensor fields and mappings which are "differentiable of class $\mathrm{C}^{\infty}$," we always omit this adjective. We deal only with connected manifolds. For the terminology concering manifolds, we follow C. Chevalley [3]. 


$$
\nabla_{X}(f \cdot Y)=f \cdot \nabla_{X} \cdot Y+(X \cdot f) \cdot Y,
$$

where $X$ and $Y$ are in $T$ and $f$ in $F^{3)} \quad \nabla_{X}$ is the so-called covariant differentiation along $X$.

The torsion tensor field $T$, of type $(1,2)$, and the curvature tensor field $R$, of type $(1,3)$, are expressed as follows:

$$
\begin{aligned}
& T(X, Y)=\nabla_{X} \cdot Y-\nabla_{Y} \cdot X-[X, Y] \\
& R(X, Y) \cdot Z=\nabla_{X} \cdot \nabla_{Y} \cdot Z-\nabla_{Y} \cdot \nabla_{X} \cdot Z-\nabla_{[X, Y]} Z,
\end{aligned}
$$

for any $X, Y$ and $Z$ in $T$ [10].

When $M$ has a Riemannian metric defined by a positive definite symmetric quadratic tensor field $G$, there is one and only one affine connection such that its torsion tensor field $T$ is zero and the covariant differentiation of the fundamental tensor field $G$ along any $X$ in $\mathfrak{I}$ is zero. This connection is called the Rjemannian connection associated to a Riemannian metric $G$.

Let $M_{i}(i=0,1, \ldots, r)$ be a manifold with an affine connection and let $M$ be the direct product of $M_{i}(i=0,1, \ldots, r)$. For any point $p=\left(p_{0}, p_{1}\right.$, $\left.\ldots, p_{r}\right)$, where $p_{i} \in M_{i}$, the mapping $s_{i}(p): p_{i}^{\prime} \rightarrow\left(p_{0}, \ldots, p_{i-1}, p_{i}^{\prime}, p_{i+1}, \ldots, p_{r}\right)$ from $M_{i}$ into $M$ defines the submanifold $M_{i}(p){ }^{4)}$ The tangent space $T(p)$ at $p$ is the direct sum of $T_{i}(p) \quad(i=0,1, \ldots, r)$, where $T_{i}(p)$ is the tangent space at $p$ of the submanifold $M_{i}(p)$. Any $X$ in $\mathfrak{I}$ can be written uniquely as a sum of $X_{i}$, where $X_{i}(p) \in T_{i}(p)$. We call $X_{i}$ the $i$-component of $X$. Moreover from a tangent vector field $X_{i}^{*}$ on $M_{i}$ we can define a tangent vector field $X_{i}: p \rightarrow d \iota_{i}(p) \cdot X_{i}^{*}$ on $M$. Let $\mathfrak{I}_{i}$ be the set of all extended tangent vector fields which are so obtained from vector fields on $M_{i}$. In the submanifold $M_{i}(p)$ through $p$, we can define a connection which is isomorphic with the given connection of $M_{i}$ by the mapping ${ }_{i}(p)$.

We can now define the product connection on $M$ as follows. For any $X$ and $Y$ in $\mathfrak{I}$

$$
\nabla_{X} \cdot Y=\sum_{i=0}^{r}\left(\nabla_{i X_{i}} \cdot Y_{i}+\sum_{i \neq j}\left[X_{i}, Y_{j}\right]_{j}\right)
$$

where the lower index $i$ (resp. $j$ ) of a vector field means the $i$ (resp. $j$ )-component and $\left(\nabla_{i X_{i}} \cdot Y_{i}\right)(p)$ is calculated on the submanifold $M_{i}(p)$ for each $i$.

3) This definition of an affine connection is due to Koszul [10].

4) Clearly two mappings $\iota_{i}(p)$ and $\iota_{i}\left(p^{\prime}\right)$ coincide if $M_{i}(p)=M_{i}\left(p^{\prime}\right)$. 
When each connection on $M_{i}$ is a Riemannian connection associated to a fundamental tensor field $G_{i}$, the product connection on $M$ is also a Riemannian connection which is associated to the product Riemannian metric $G$.

For any $p \in M, M_{i}(p)(i=0,1, \ldots, r)$ is a totally geodesic submanifold, that is, any geodesic curve tangent to $M_{i}(p)$ at a point is contained in $M_{i}(p)$.

Let $T_{i}$ and $T$ be the torsion tensor fields of $M_{i}$ and $M$ respectively, and $R_{i}$ and $R$ the curvature tensor fields of $M_{i}$ and $M$ respectively. Then it is easily seen that

$$
\begin{aligned}
& T(X, Y)=\sum_{i=0}^{r} T_{i}\left(X_{i}, Y_{i}\right) \\
& R(X, Y) Z=\sum_{i=0}^{r} R_{i}\left(X_{i}, Y_{i}\right) Z_{i},
\end{aligned}
$$

where $\left(T_{i}\left(X_{i}, Y_{i}\right)\right)(p)$ and $\left(R_{i}\left(X_{i}, Y_{i}\right) Z_{i}\right)(p)$ are calculated in $M_{i}(p)$. Indeed, $T$ and $R$ being tensors, the above equalities have only to be verified for $X_{i} \in \mathfrak{I}_{i}, Y_{j} \in \mathfrak{I}_{j}$ and $Z_{k} \in \mathfrak{I}_{k}$.

2. Any tangent vector field $V$ on a manifold $M$ generates a local oneparameter group of local transformations $\varphi_{t}(-\varepsilon<t<\varepsilon)^{5)}$ such that

$$
\lim _{t \rightarrow 0} \frac{1}{t}\left(\varphi_{t} \circ f-f\right)=V \cdot f,
$$

for any $f$ in $\tilde{F}$. A vector field $V$ on $M$ with an affine connection is called an infinitesimal affine transformation if it generates a local one-parameter group of local affine transformations, that is,

$$
d \varphi_{t}\left(\nabla_{X} \cdot Y\right)=\Delta_{d_{\vartheta} t(X)} \cdot\left(d \varphi_{t}(Y)\right)
$$

for any $X$ and $Y$ in $\mathfrak{I}$, and for any $t(-\varepsilon<t<\varepsilon)$. When $M$ has a Riemannian metric, we say that $V$ is a Killing vector field if it generates a local oneparameter group of local isometries, that is,

$$
G\left(d \varphi_{t}(X), d \varphi_{t}(Y)\right)=G(X, Y)
$$

for any $X$ and $Y$ in $\mathfrak{I}$, and for any $t(-\varepsilon<t<\varepsilon)$.

A necessary and sufficient condition for a vector field $V$ to be an infinitesimal affine transformation is that we have

5) More precisely, for each point $p$, there exist a neighbourhood $U(p)$ and a positive number $\varepsilon$ such that $\phi_{t}(-\varepsilon<t<\varepsilon)$ defined on $U(p)$. 
(6)

$\nabla_{X} \cdot \nabla_{Y} \cdot(V)-\nabla_{\nabla_{X} \cdot Y} \cdot(V)+\nabla_{X} \cdot(T(V, Y))-T\left(V, \nabla_{X} \cdot Y\right)+R(V, X) Y=0$

for any $X$ and $Y$ in $\mathfrak{T}$. Using the covariant differentiation and the contraction, we can write the above equality as follows:

$$
\left\langle\nabla \nabla V, Y \otimes X>+\left(\nabla_{X} \cdot T\right)(V, Y)+T\left(\nabla_{X} \cdot V, Y\right)+R(V, X) Y=0 .\right.
$$

A vector field $V$ is a Killing vector field if and only if it satisfies

$$
G\left(\nabla_{X} \cdot V, Y\right)+G\left(X, \nabla_{Y} \cdot V\right)=0
$$

for any $X$ and $Y$ in $\mathfrak{T}$. The equalites (6) and (7) can be obtained by computing the Lie derivatives along $V$ of the both sides of (4) and (5) respectively.

Lemma 1. Let $M=M_{0} \times \ldots \times M_{r}$ be a product manifold with a product affine connection, and let $V$ be an infinitesimal affine transformation on $M$ with the i-component $V_{i}$. Then $V_{i}$ is an infinitesimal affine transformation on $M$ and the restriction of $V_{i}$ on $M_{i}(p)$ is an infinitesimal affine transformation on $M_{i}(p)$ for each $i$.

Proof. From (1), for a vector $Z$ such that $Z(p) \in T_{i}(p)$ at each point, we have

$$
\left(\nabla_{X} \cdot Z_{i}\right)(p) \in T_{i}(p),
$$

for any $X \in \mathfrak{I}$. From (2) and (3) we have

$$
(T(V, X))_{i}=T\left(V_{i}, X\right) \quad \text { and } \quad(R(V, X) Y)_{i}=R\left(V_{i}, X\right) Y,
$$

for any $X$ and $Y$ in $\mathfrak{I}$. The $i$-component of the left hand side of (6) is equal to the left hand side of (6) in which $V$ is replaced by $V_{i}$. This shows that $V_{i}$ is an infinitesimal affine transformation on $M$.

Next, from (1) we can see easily

$$
\nabla_{X_{i}} \cdot Y_{i}=\nabla_{i X_{i}} \cdot Y
$$

and this vector field is contained in $\mathfrak{I}_{i}$ for $X_{i}$ and $Y_{i}$ in $\mathfrak{I}_{i}$. Moreover for a vector field $Z$ such that $Z(p) \in T_{i}(p)$ at each point $p$, we have

$$
\nabla_{X_{i}} \cdot Z=\nabla_{i x_{i}} \cdot Z
$$

for $X_{i} \in \mathfrak{I}_{i} . \quad$ From (2) and (3) it follows that

$$
\begin{aligned}
& (T(X, Y))_{i}=T_{i}\left(X_{i}, Y_{i}\right) \\
& (R(X, Y) \cdot Z)_{i}=R_{i}\left(X_{i}, Y_{i}^{\prime}\right) \cdot Z_{i},
\end{aligned}
$$


for any $X$ and $Y$ in $\mathfrak{I}$. Therefore, the $i$-component of the left hand side of (6) for $X=X_{i}$ in $\mathfrak{I}_{i}$ and $Y=Y_{i}$ in $\mathfrak{I}_{i}$ is equal to

$$
\begin{aligned}
\nabla_{i X_{i}} \cdot \nabla_{i Y_{i}} \cdot\left(V_{i}\right) & +\nabla_{i \nabla_{i X_{i}} \cdot Y_{i}} \cdot\left(V_{i}\right)+\nabla_{i X_{i}} \cdot\left(T_{i}\left(V_{i}, Y_{i}\right)\right) \\
& -T_{i}\left(V_{i}, \nabla_{i X_{i}} \cdot\left(Y_{i}\right)\right)+R_{i}\left(V_{i}, X_{i}\right) \cdot Y_{i} .
\end{aligned}
$$

Since each term is calculated on $M_{i}(p)$, this shows that the restriction of $V$ on $M_{i}(p)$ is an infinitesimal affine transformation on $M_{i}(p)$.

When $V$ is a Killing vector field the situation is just the same as above.

Remark. It is almost evident that the vector field $V_{i}$ in $\mathfrak{I}_{i}$ which is the extended vector field of an infinitesimal affine transformation (resp. a Killing vector field) of $M_{i}$ is also an infinitesimal affine transformation (resp. a Killing vector field) of $M$.

We say that a manifold with an affine connection (or a Riemannian manifold) $M$ is complete if every geodesic curve can be extended for any large value of the canonical parameter. When the completeness is satisfied on $M$, any infinitesimal affine transformation (or a Killing vector field) generates a one-parameter group of affine transformations (isometries) from $M$ onto itself [7].

3. Let $M$ be a manifold with an affine connection. The group $A(M)$ of all affine transformation of $M$ onto itself is a Lie group with respect to the compact-open topology [5], [6], [9]. When $M$ has a Riemannian metric, the group $I(M)$ of all isometries of $M$ onto itself is a closed subgroup of $A(M)$. $I(M)$ is also a Lie group [8].

The mapping from $A(M) \times M$ onto $M$, which gives the transformation law, is differentiable as is known from a theorem of S. Bochner and D. Montgomery [1]. Any one-parameter subgroup in $A(M)$ (resp. $I(M)$ ) induces an infinitesimal affine transformation (resp. a Killing vector field) on $M$.

\section{Decomposition Theorem of $A_{0}(M)$ and $I_{0}(M)$}

4. In this section we always assume that $M$ is a simply connected complete Riemannian manifold.

The homogeneous holonomy group $\Psi(p)$ at $p$ operates on the tangent space $T(p)$ and is completely reducible. If $T(p)$ is irreducible, we call $M$ irreducible.

The following theorem is due to de Rham [4]: 
1) A simply connected complete Riemannian manifold $M$ is isometric to the direct product of a Euclidean space $M_{0}$ and simply connected complete irreducible Riemannian manifolds $M_{i}$ with $\operatorname{dim} M_{i} \geqslant 2(i=1, \ldots, r)$, and this decomposition is unique up to the order and isometries.

2) The tangent space $T(p)$ at each point $p$ is the direct sum of the $\Psi(p)$-invariant subspace $T_{0}(p), T_{1}(p), \ldots, T_{r}(\not p)$, where $T_{0}(\not p)$ is the subspace of all $\Psi(p)$-invariant vectors, $T_{i}(p)(i=1, \ldots, r)$ are irreducible and any two subspaces $T_{i}(p)$ and $T_{j}(p)(i \neq j, i, j=0,1, \ldots, r)$ are mutually orthogonal. This decomposition is unique.

If we identify $M$ with the direct product $M_{0} \times M_{1} \times \ldots \times M_{r}$, the subspace $T_{i}(p)$ is the tangent space at $p$ of the submanifold $M_{i}(p)$ which corresponds to $M_{i}$ in the manner mentioned in 1 .

We call these decompositions the de Rham decomposition of the manifold, or of the tangent space $T(p)$.

5. The aim of this section is to prove the following

THEOREM 1. Let $M$ be a simply connected complete Riemannian rsanifold, and $M=M_{0} \times M_{1} \times \ldots \times M_{r}$ be the de Rham decomposition of $M$. Then the group $A_{0}(M)$ is isomorphic to the direct product $A_{0}\left(M_{0}\right) \times A_{0}\left(M_{1}\right) \times \ldots$ $\times A_{0}\left(M_{r}\right)$, and the group $I_{0}(M)$ is isomorphic to the direct product $I_{n}\left(M_{0}\right)$ $\times I_{0}\left(M_{1}\right) \times \ldots \times I_{0}\left(M_{r}\right)$, where $A_{0}(M)$ and $A_{0}\left(M_{i}\right)$ (resp. $I_{0}(M)$ and $I_{0}\left(M_{i}\right)$ ) are the connected components of the identity in $A(M)$ and $A\left(M_{i}\right)$ (resp. $I(M)$ and $\left.I\left(M_{i}\right)\right)$ respectively.

To prove this theorem the following lemma, which is given by K. Nomizu $[11],[12]$ is useful.

Lemma 2. Let $T(p)=T_{n}(p)+T_{1}(p)+\ldots+T_{r}(p)$ be the de Rham decomposition of the tangent space. Then

$$
d \varphi\left(T_{i}(p)\right)=T_{i}(\varphi(p)),
$$

for any $\varphi \in A_{0}(p)$ and for any $p \in M$.

Proof of Theorem 1. Let $\varphi_{i}$ be an element in $A_{0}\left(M_{i}\right)(i=0,1, \ldots, r)$, a transformation $\varphi(p)=\left(\varphi_{0}\left(p_{0}\right), \ldots, \varphi_{i}\left(p_{i}\right), \ldots, \varphi_{r}\left(p_{r}\right)\right)$, where $p=\left(p_{0}, \ldots\right.$, $\left.p_{i}, \ldots, p_{r}\right)$ is an element in $A_{0}(M)$. The mapping from $A_{0}\left(M_{0}\right) \times A_{0}\left(M_{1}\right)$ $\times \ldots \times A_{0}\left(M_{r}\right)$ into $A_{0}(M)$ which maps $\left(\varphi_{0}, \varphi_{1}, \ldots, \varphi_{r}\right)$ to $\varphi$ is an isomorphism. Then to prove the theorem, we have only to show that the induced 
isomorphism from the direct sum of the Lie algebras associated to $A_{0}\left(M_{i}\right)$ $(i=0,1, \ldots, r)$ into the Lie algebras a associated to $A_{0}(M)$ is onto. The Lie algebra $\mathfrak{a}$ is the Lie algebra of all infinitesimal affine transformations on $M$. The set $\mathfrak{a}_{i}$ of all infinitesimal affine trasformations contained in $\mathfrak{I}_{i}$, namely the image of the Lie algebra associated to $A_{0}\left(M_{i}\right)$ by the induced isomorphism, is an ideal for each $i$.

Let $V$ be an infinitesimal affine transformation in $\mathfrak{a}$ and $V_{i}$ the $i$-component of $V$. We now fix an arbitrary point $p^{*}=\left(p_{0}^{*}, \ldots, p_{i}^{*}, \ldots, p_{r}^{*}\right)$ and consider the mapping $c_{i}\left(p^{*}\right)$ from $M_{i}$ onto $M_{i}\left(p^{*}\right)$ as stated in 2 . From Lemma $1 V_{i}$ and the restriction of $V_{i}$ on $M_{i}\left(p^{*}\right)$ are infinitesimal affine transformations on $M$ and $M_{i}\left(p^{*}\right)$ respectively, and there is an infinitesimal affine transformation $V_{i}^{\prime}$ on $M_{i}$ such that $d_{i}\left(p^{*}\right)\left(V_{i}^{\prime}\right)$ is equal to the restriction of $V_{i}$ on $M_{i}\left(p^{*}\right)$. Let $V_{i}^{*}$ be the infinitesimal affine transformation on $M$ which is the extension of $V_{i}^{\prime}$. Clearly $V_{i}^{*}$ is contained in $\mathfrak{I}_{i}$. We shall prove that $V_{i}^{*}=V_{i}$.

We now consider the difference $V_{i}-V_{i}^{*}$. Clearly it is an infinitesimal affine transformation on $M$, and $\left(V_{i}-V_{i}^{*}\right)(p)$ is zero when $p$ is on $M_{i}\left(p^{*}\right)$. From our assumption that $M$ is complete, $V_{i}-V_{i}^{*}$ generates a one-parameter subgroup $\varphi_{t}$ in $A_{0}(M)$. First, as $\left(V_{i}-V_{i}^{*}\right)(p)=0$ on $M_{i}\left(p^{*}\right)$, each transformation $\varphi_{t}$ leaves every point on $M_{i}\left(p^{*}\right)$ fixed. And as $\left(V_{i}-V_{i}^{*}\right)\left(p^{*}\right) \in T_{i}(p)$ at any point on $M$, we have $\varphi_{t}(p) \in M_{i}(p)$, namely, each $\varphi_{t}$ maps $M_{i}(p)$ onto itself. Next we consider the submanifold $M_{i}^{\wedge}(p)=\left\{p^{\prime} ; p^{\prime} \in M, p^{\prime}=\left(p_{0}^{\prime}, \ldots\right.\right.$, $\left.p_{i-1}^{\prime}, p_{i}, p_{i+1}^{\prime}, \ldots, p_{r}^{\prime}\right)$, where $\left.p_{j}^{\prime} \in M_{j}(j \neq i)\right\}$ through $p=\left(p_{0}^{*}, \ldots, p_{i-1}^{*}, p_{i}\right.$, $\left.p_{i+1}^{*}, \ldots, p_{r}^{*}\right)$ which is on $M_{i}\left(p^{*}\right)$. The tangent space of $M_{\hat{i}}(p)$ at $p^{\prime}$ is $T_{i}\left(p^{\prime}\right)$ $=T_{0}\left(p^{\prime}\right)+\ldots+T_{i-1}\left(p^{\prime}\right)+T_{i+1}\left(p^{\prime}\right)+\ldots+T_{r}\left(p^{\prime}\right)$. Let $q$ be an arbitrary point on $M_{\hat{i}}(p)$. As $M_{\hat{i}}(\not p)$ is a totally geodesic submanifold and is complete, $p$ and $q$ can be joined by a geodesic curve $\sigma(s)$ on $M_{\hat{i}}(p)$, and the tangent vector $X(p)$ of $\sigma(s)$ at $p$ is contained in $T \hat{i}(p)$. According to Lemma 2, we have $d \varphi_{t} \cdot X(p) \in T \hat{i}(p)$ for any real number $t$, and $d \varphi_{t} \cdot X(p)$ is the tangent vector of a geodesic curve $\varphi_{t} \cdot \sigma(s)$ at $p$. Therefore the geodesic curve $\varphi_{t} \cdot \sigma(s)$ lies on $M_{\hat{i}}(p)$, and $\varphi_{t}(q)$ is in $M_{\hat{i}}(p)$. This shows $\varphi_{t}\left(M_{\hat{i}}(p)\right) \subset M_{\hat{i}}(p)$ for any $p$ on $M_{i}\left(p^{*}\right)$. Any point $q$ on $M$ is contained in one and only one $M_{\hat{i}}(p)$ through some $p$ on $M_{\hat{i}}\left(p^{*}\right)$ and, on the other hand, in $M_{i}(q)$. Hence $\varphi_{t}(q)$ $\in M_{\hat{i}}(p) \frown M_{i}(q)$. But $M_{i}(q)$ and $M_{\hat{i}}(p)$ have one and only one common point $q$, and hence $\varphi_{t}(q)=q$. Thus we conclude that $\varphi_{t}$ is the identity transfor- 
mation for any $t$ and $V_{i}=V_{i}^{*}$ on $M$. This shows that any element $V$ in a can be written as a sum of elements in $\mathfrak{a}_{i}(i=0,1, \ldots, r)$, and our isomorphism is onto.

\section{An Application of the Decomposition Theorem}

6. Let $M$ be a Riemannian manifold with the fundamental tensor field $G$. For any $\varphi$ in $A(M), d \varphi \cdot G$ is also a positive definite symmetric tensor field and as $d \varphi$ is commutative with the covariant differentiation, $\nabla(d \varphi \cdot G)=0$. Then $(d \varphi \cdot G)(p)$ is invariant by the operations of the homogeneous holonomy group $\Psi(p)$. If $M$ is irreducible, there is a positive constant $c(\varphi)$ such that $d \varphi \cdot G$ $=c^{2}(\varphi) \cdot G$, and $\varphi \rightarrow c(\varphi)$ is a continuous representation of $A(M)$ into the multiplicative group of all positive real numbers [11], [12]. This follows from the fact that, the invariant positive definite bilinear form on $T(p)$ is uniquely determined up to a positive constant, as the homogeneous holonomy group is irreducible. From this fact the following lemma is easily obtained.

LemMa 3. When a Riemannian manifold $M$ is irreducible and complete, an infinitesimal affine transformation $V$ is a Killing vector field if and only lf there is a non-trivial orbit on which the length of $V$ is bounded.

Proof. Let $\varphi_{t}$ be the one-parameter group generated by $V$. Then $V\left(\varphi_{t}(p)\right)$ $=d \varphi_{t} \cdot V(p)$ and $\left|V\left(\varphi_{t}(p)\right)\right|=c(\varphi)|V(p)|$, where $|X(p)|$ denotes the length of a vector $X(p)$. If $V$ is a Killing vector field, $c\left(\varphi_{t}\right)=1$ for every $t$ and surely the length of $V$ is bounded on any orbit $\left\{\varphi_{t}(p)\right\}$. Conversely, let $\left\{\varphi_{t}(p)\right\}$ be a non-trivial orbit on which the length of $V$ is bounded. Then $V(p)$ is not zero and the function $c\left(\varphi_{t}\right)$ of $t$ is bounded. As already mentioned, $c\left(\varphi_{t}\right)$ is a continuous representation of the additive group of all real numbers. Therefore $c\left(\varphi_{t}\right)$ must be equal to 1 for every $t$, and $V$ is a Killing vector field.

7. A Riemannian manifold is called locally flat when the homogeneous holonomy group is discrete.

If there is an absolutely parallel vector field ${ }^{6)}$ on a complete Riemannian manifold, it is a Killing vector field. We define a translation as an isometry which lies on the one-parameter group of isometries generated by such a Killing

6) A vector field $X$ is an absolutely parallel vector field when for any two points $p$ and $q$, $X(p)$ and $X(q)$ are parallel along any piece-wise differentiable curve which joins $p$ and $q$. This condition is equivalent to the condition $\nabla X=0$. 
vector field.

For the necessity in the next section we prepare

LEMMA 4. If the length of an infinitesimal affine transformation $V$ on a complete locally flat Riemannian manifold is bounded, then $V$ is a Killing vector field and generates a one-parameter group of translations.

Proof. The universal covering manifold $\tilde{M}$ of $M$ has a natural Riemannian metric induced from that of $M$, with respect to which $\tilde{M}$ is also complete and locally flat. As is well known, ${ }^{7)}$ a complete locally flat Riemannian manifold $M$ is isometric to a Euclidean space with the usual metric when it is simply connected, and the group of all affine transformations $A(\tilde{M})$ is the usual affine group. If we take a system of cartesian coordinates $x^{1}, x^{2}, \ldots, x^{n}$ on $\tilde{M}$, then an infinitesimal affine transformation $\widetilde{V}$ is given by

$$
\sum_{i=1}^{n}\left(\sum_{j=1}^{n} a_{i j} \cdot x^{j}+b_{i}\right) \frac{\partial}{\partial x^{i}}
$$

where $a_{i j}$ and $b_{i}$ are arbitrary real numbers. It is easily seen that the length $\sqrt{\sum_{i=1}^{n}\left(\sum_{j=1}^{n} a_{i j} \cdot x^{j}+b_{i}\right)}$ of $V$ is bounded on $M$ if and only if each $a_{i j}(i, j=1,2$, $\ldots, n)$ is zero.

For a Killing vector field $V$ on $M$ whose length is bounded, there is a Killing vector field $\tilde{V}$ on $\tilde{M}$ such that the projection from $\tilde{M}$ to $M$ maps $\tilde{V}$ on $V$, and its length is bounded on $M$. Then $\widetilde{V}$ and accordingly $V$ are absolutely parallel vector fields on $\tilde{M}$ and $M$ respectively.

Corollary. If $M$ is a compact locally flat Riemannian manifold, $A_{0}(M)$ coincies with $I_{0}(M)$ and is abelian.

8. THEOREM 2. Let $M$ be a complete Riemannian manifold. If the lengih of an infinitesimal affine transformation $V$ is bounded on $M$, then $V$ is a Killing vector field.

Proof. Let $\tilde{M}$ be the universal covering manifold of $M$ and let $\tilde{M}$ $=\tilde{M}_{0} \times \tilde{M}_{1} \times \ldots \times \tilde{M}_{r}$ be the de Rham decomposition of $M$. It has a Riemannian connection which is naturally induced from that of $M$, and is complete with respect to it. If $\tilde{V}$ is the infinitesimal affine transformation

7) For example, cf. [2] Chapitre III, II. 
which is mapped on $V$ by the projection from $\tilde{M}$ onto $M$, then the length of $\widetilde{V}$ is also bounded on $\tilde{M}$. As $\tilde{M}$ is simply connected and complete, we can apply the arguments in II.

Since the length of $\tilde{V}$ is bounded on $\tilde{M}$, the length of the $i$-component $\tilde{V}_{i}$ of $\tilde{V}$ is also bounded on $\tilde{M}$. As we have seen in the proof of Theorem 1 , $\tilde{V}_{i}$ is the extension of a certain infinitesimal affine transformation $\tilde{V}_{i}^{\prime}$ on $\tilde{M}_{i}$, and the length of $\tilde{V}_{i}^{\prime}$ is clearly bounded. We see that $\tilde{V}_{i}^{\prime}$ is a Killing vector field from Lemma 3 when $1 \leqq i \leqq r$, and from Lemma 4 when $i=0$. As we already remarked, the extension $\tilde{V}_{i}$ of a Killing vector field $\tilde{V}_{i}^{\prime}$ on $\tilde{M}_{i}$ is also a Killing vector field on $\tilde{M}$ for each $i$. Hence $\tilde{V}$ and accordingly $V$ are Killing vector fields on $\tilde{M}$ and $M$ respectively.

On a compact Riemannian manifold, the length of any vector field is of course bounded. Hence we have

Theorem 3. (K. Yano) On a compact Riemannian manifold $M$, every infinitesimal affine transformation is a Killing vector field. Therefore $A_{0}(M)$ coincides with $I_{\mathrm{c}}(M)$.

\section{BiBLIOGRAPHY}

[1] S. Bochner and D. Montgomery: Groups of differentiable and real or complex analytic transformations, Ann. of Math., vol. 46 (1945), pp. 685-694.

[2] E. Cartan: Leçons sur la géométrie des espaces de Riemann, Paris, (1946).

[ 3 ] C. Chevalley: Theory of Lie groups I, Princeton University Press, (1946).

[4] G. de Rham: Sur la réductibilité d'un espace de Riemann, Comm. Math. Helv., vol. 26 (1952), pp. 328-344.

[5] J. Hano and A. Morimoto: Note on the group of affine transformations of an affinely connected manifold, Nagoya Math. Jour., vol. 8 (1955), pp. 85-95.

[6] S. Kobayashi: Groupe de transformations qui laissent invariante une connexion infinitésimale, Comptes rendus, 238 (1954), pp. 644-645.

[7] S. Kobayashi : Espaces à connexion de Cartan complets, Proc. of the Jap. Acad., vol. 30 (1953), pp. 709-710.

[8] S. Myers and N. Steenrod: The group of isometries of a Riemannian manifold, Ann. of Math., vol. 40 (1939), pp. 400-416.

[9] K. Nomizu: On the group of affine transformations of an affinely connected manifold, Proc. of the Amer. Math. Soc., vol. 4 (1953), pp. 816-823.

[10] K. Nomizu: Invariant affine connections on homogeneous spaces, Amer. Jour. of Math., vol. 76 (1954), pp. 33-65. 
[11] K. Nomizu: Sur les transformations affines d'une variété riemannienne, Comptes rendus, 237 (1953), pp. 1308-1310.

[12] K. Nomizu: Studies on Riemannian homogeneous spaces, In this Journal.

[13] K. Yano: On harmonic and Killing vector fields, Ann. of Math., vol. 55 (1952), pp. $38-45$.

Mathematical Institute,

Nagoya University 